\title{
Entrelacs
}

Cinéma et audiovisuel

\section{Scénariser le rôle et le pouvoir d'agir de l'utilisateur : vers une typologie interactionnelle du documentaire interactif}

\section{Samuel Gantier}

\section{(2) OpenEdition \\ Journals}

Electronic version

URL: http://journals.openedition.org/entrelacs/1840

DOI: $10.4000 /$ entrelacs. 1840

ISSN: 2261-5482

Publisher

Éditions Téraèdre

\section{Electronic reference}

Samuel Gantier, «Scénariser le rôle et le pouvoir d'agir de l'utilisateur : vers une typologie interactionnelle du documentaire interactif », Entrelacs [Online], 12 | 2016, Online since 19 April 2016, connection on 19 April 2019. URL : http://journals.openedition.org/entrelacs/1840 ; DOI : 10.4000/ entrelacs. 1840

This text was automatically generated on 19 April 2019.

Tous droits réservés 


\title{
Scénariser le rôle et le pouvoir d'agir de l'utilisateur : vers une typologie interactionnelle du documentaire interactif
}

\author{
Samuel Gantier
}

1 Sur la décennie 2005-2015, près de quatre cents web-documentaires francophones ont été publiés sur Internet. Une cartographie des principaux acteurs de la production (Gantier, 2012) identifie deux logiques de conception symétriquement opposées. D'une part, des outils d'aide à la conception d'origine française $\left(\mathrm{Klynt}^{1}, 3 \mathrm{WDOC}^{2}\right.$, RacontR ${ }^{3}$ ) favorisent la publication de récits hypermédias sans qu'aucune expertise en développement web ne soit nécessaire ${ }^{4}$. S'il est exact que ces outils permettent de rationaliser les coûts de production - en proposant notamment aux concepteurs une série de ressources (gabarits, bibliothèques graphiques) - il n'en est pas moins vrai que ces véritables 《 architextes $^{5}$ » (Jeanneret et Souchier, 1999) conditionnent les potentialités créatives du projet (cf. Bolka-Tabary et Gantier, 2011). D'autre part, des œuvres innovantes, plus ambitieuses et plus coûteuses sont régulièrement produites sous l'impulsion des fonds étatiques et régionaux d'aide aux «nouveaux médias " ${ }^{6}$.

Dans ce contexte, l'internaute doit s'acculturer à des «interface-films » (Di Crosta, 2009) qui mélangent les codes filmiques du genre documentaire aux modes d'interaction propres aux « médias informatisés » (Jeanneret, 2007). Cette nouvelle praxis documentaire modifie en profondeur la place et le rôle octroyé à un spectateur-actant. Le paradigme auctorial du "point de vue documenté » cher à Jean Vigo est ainsi reformulé à travers une scénarisation de l'interactivité. Dès lors, selon quels critères peut-on classer l'hétérogénéité de ces écritures documentaires interactives? Quelles sont les spécificités communicationnelles de ce format hybride et non stabilisé ? De quelle manière et sous quelles conditions ces dispositifs médiatiques proposent-ils de nouvelles formes relationnelles de re-présentation du réel? 
3 L'approche interactionnelle adoptée dans cet article propose d'expliciter la posture spectatorielle qui caractérise le format web-documentaire. Pour ce faire, une première partie expose les enjeux épistémologiques sous-jacents à la notion polysémique d'interactivité. Dans la continuité des recherches menées dans le champ de l'audiovisuel interactif (Perény et Amato, 2011), de la fiction interactive (Di Crosta, 2009), du Net art (Fourmentraux, 2010) et de la littérature numérique (Bouchardon, 2009), l'axe de pertinence retenu vise à qualifier les opérations textuelles attendues de la part de l'utilisateur. Dans un deuxième temps, cette étude propose de reformuler la typologie des modes d'interaction établie par Sandra Gaudenzi (2013). L'objectif consiste à inventorier l'espace hétérogène du documentaire interactif suivant six entrées complémentaires: hypermédiatique, dialogique, contributif, participatif, ludique et performatif. Sur la base d'exemples emblématiques de la production, cette catégorisation interroge le processus de médiation entre dispositif numérique et narration du «réel ». En d'autres termes, l'enjeu consiste à questionner la relation systémique entre quatre entités : l'instance d'énonciation, le réel, le dispositif médiatique et l'interacteur. L'ambition heuristique de cette typologie est donc double : elle permet d'une part de discerner les spécificités du travail de conception et d'autre part d'analyser les compétences spectatorielles mobilisées par l'instance de réception.

\section{Enjeux terminologiques de la notion d'interactivité}

4 S'il va de soi que tout média informatisé possède une dimension interactive, le fait de qualifier d'interactif le format web-documentaire relève d'un truisme qui énonce la réalité la plus élémentaire de son fonctionnement. Depuis l'avènement de la télématique en France au cours de années $1980^{7}$, un foisonnement terminologique associe la notion d'interactivité aux différentes possibilités d'agir sur un programme ou un dispositif médiatique. La revue de littérature établie par Jean-Thierry Julia (2003) dans le champ des sciences de l'information et de la communication, de l'éducation et de l'informatique analyse la diffusion de ce terme. De la "pédagogie active " à l'innovation de dispositifs socio-techniques en passant par la réception des médias, la problématique récurrente revient à définir la place et l'activité que ces objets dits interactifs attribuent à l'instance de réception. Chaque acception propose ainsi de préciser les types d'actions et les modalités d'intervention offertes à l'utilisateur dans le système. De manière synthétique, l'analyse comparative menée par Julia met en exergue quatre acceptions principales ${ }^{8}$ :

5 - Premièrement, de nombreuses dénominations métonymiques visent à assimiler l'enchâssement des dispositifs médiatiques comme un tout. Ainsi, lorsqu'une dimension du système est vérifiée interactive, un méta-objet qui recouvre l'objet initial sera également considéré interactif par effet de contagion. À titre d'exemple, l'interactivité de vote revendiquée par certains programmes télévisés n'est envisageable que par amalgame avec d'autres dispositifs médiatiques (télématique, téléphonie, Web, réseaux sociaux numériques, etc.).

6 - Deuxièmement, l'interactivité homme-machine est fréquemment présentée comme la simulation d'un dialogue humain. Or, ce qui caractérise les modes de communication interhumains c'est précisément "leur capacité à faire avec leurs ignorances, incompréhensions, incertitudes, approximations que, pour l'essentiel, l'approche informatique s'épuise à faire disparaitre » (Balpe, 1990 : 162). Autrement dit, pour qu'un système interactif apparaisse comme réellement interactif, il faudrait qu'il puisse 
«prendre en compte ce degré de liberté, d'incertitude interprétative caractéristique des mécanismes humains de transmission du sens » (ibid.).

7 - Troisièmement, des acceptions bipolaires de la notion d'interactivité opposent, d'une part, une vision ergonomique et utilitaire des échanges d'information avec la machine, et d'autre part l'engagement de l'instance d'énonciation du document avec l'utilisateur final. Ces approches prennent différents vocables selon les contextes historiques de l'étude: interactivité «fonctionnelle vs intentionnelle»; «mécanique» vs significative »; « d'usage vs de contenu »; « transitive vs intransitive ».

8 - Enfin, une quatrième approche tente de définir des modalités d'action de l'utilisateur. Le degré d'interactivité d'un document revient ici à désigner l'acte d'interprétation du lecteur face au texte ${ }^{9}$. La problématique de l'interactivité amène sous ce prisme à discuter de la confrontation entre "ergonomie fonctionnelle [et] herméneutique de la lecture » (Jeanneret, $2007: 170$ ).

$9 \mathrm{Au}$ vu de ce rapide état de l'art, force est de constater, dans la lignée de Catherine Guéneau (2005), qu'une définition théorique générale et définitive de la notion d'interactivité est introuvable. Afin de contourner cette aporie, une approche circonscrite à un contexte pragmatique de lecture-écriture du web-documentaire a donc été adoptée dans le cadre de cette étude. L'enjeu consiste dès lors à qualifier la posture spectatorielle ainsi que l'action et la place de l'utilisateur au sein du dispositif proposé par le documentaire interactif. Ainsi, comme le démontre Étienne Perény et Étienne Armand Amato (2011), dans une perspective historique relevant de l'intermédialité, la nature spectatorielle du flux audiovisuel interactif est orientée selon les spécificités médiatiques propres à la télévision, au Web ou aux jeux vidéo. Cette approche met en exergue une tension entre une logique réticulaire et un mode d'intervention séquentiel. Le webdocumentaire, en tant qu'hypermédia, serait clivé entre le modèle médiatique de la télévision (filiation originale) et le modèle du jeu vidéo (idéal du média informatisé). Suivant ce raisonnement, la logique relationnelle qui définit l'accès aux données est tendu par une dialectique entre une consultation de type "réticulaire» et une immersion "véhiculaire ». Le mode de lecture qui en résulte alterne des actions de manipulation sur l'interface et l'immersion dans un flux audiovisuel, comme le synthétise le tableau cidessous. 


\begin{tabular}{|c|c|c|c|}
\hline & Télévision & Web & Jeu vidéo \\
\hline $\begin{array}{l}\text { Nature } \\
\text { médiatique }\end{array}$ & Mass-média & Hypermédia & Cybermédia \\
\hline $\begin{array}{l}\text { Logique } \\
\text { relationnelle }\end{array}$ & Tentaculaire & Réticulaire & Véhiculaire \\
\hline $\begin{array}{l}\text { Régime } \\
\text { d'audio-vision }\end{array}$ & Exposition & $\begin{array}{l}\text { Visualisation, } \\
\text { Visionnage }\end{array}$ & Immersion \\
\hline $\begin{array}{l}\text { Régime } \\
\text { d'interactivité }\end{array}$ & $\begin{array}{l}\text { Sélection (zapping), } \\
\text { voire votes en retour }\end{array}$ & $\begin{array}{l}\text { Recherche, } \\
\text { Consultation, } \\
\text { Manipulation, } \\
\text { Commentaires }\end{array}$ & $\begin{array}{l}\text { Accomplissement } \\
\text { d'une tâche }\end{array}$ \\
\hline $\begin{array}{l}\text { Mode } \\
\text { d'intervention }\end{array}$ & Ponctuel & Séquentiel & Couplé \\
\hline $\begin{array}{l}\text { Spatio- } \\
\text { temporalité }\end{array}$ & $\begin{array}{l}\text { Simultanéité } \\
\text { collective, avec } \\
\text { distance annulée ou } \\
\text { visionnage différé de } \\
\text { documents temporels } \\
\text { (films, vidéos) }\end{array}$ & $\begin{array}{l}\text { Espaces fragmentaires } \\
\text { reliés, avec accès } \\
\text { immédiat aux documents } \\
\text { temporels ou à des } \\
\text { espaces simulés (jeux } \\
\text { vidéo, univers } \\
\text { persistants) }\end{array}$ & $\begin{array}{l}\text { Espace imaginaire } \\
\text { simulé. } \\
\text { Temporalité } \\
\text { continue, avec } \\
\text { exploration des } \\
\text { possibles narratifs } \\
\text { et existentiels }\end{array}$ \\
\hline
\end{tabular}

Tableau : Crible analytique de l'audiovisuel interactif (Perény et Amato, 2011 : 33).

En outre, les recherches de Marida Di Crosta (2009) sur le cinéma interactif mettent en exergue la posture méta-narrative de l'interacteur qui peut désormais intervenir sur le déroulement du récit. Suivant cette optique, le projet du cinéma interactif réside dans le questionnement de l'interactivité comme un «critère fondateur de la relation du spectateur au film (voire des spectateurs entre eux), comme une nouvelle forme d'interdépendance relationnelle de l'auteur et du récepteur vis-à-vis de la construction de l'expérience et du contenu narratif, visuel et sonore » (Di Crosta, 2009 : 13). Au-delà des voies qu'elle ouvre pour la création, l'apport heuristique de cette recherche est d'argumenter le caractère fondamentalement séquentiel de ce type de consultation. Marida Di Crosta parle ainsi de "double registre de narration" pour souligner l'alternance des événements automatiques et ceux générés par le spectateur. Il s'agit d'un processus binaire qui alterne l'immersion dans des séquences en continu, l'interruption du flux pour permettre un épisode interactif puis la reprise du flux. Vue sous ce prisme, la posture spectatorielle du web-documentaire résulte d'une tension entre immersion et intervention dans la narration. En d'autres termes, l'interacteur peut soit intervenir en orientant son parcours de lecture, soit opérer une suspension de la narration en consultant des documents annexes. Dans le champ de la littérature numérique, Serge Bouchardon précise cette problématique en soulignant que les actions du lecteur sur le récit rejouent le rapport classique «adhésion-distanciation». En résumé, la problématique du récit littéraire interactif ${ }^{10}$ consiste à déterminer s'il faut conduire ou faire conduire le récit. En effet, « la narrativité consiste à prendre le lecteur par la main pour lui raconter une histoire, du début à la fin. L'interactivité, quant à elle, consiste à donner la main au lecteur, qui devient ainsi interacteur, pour intervenir au cours du récit, et cela à différents niveaux (histoire, structure du récit, narration) " (Bouchardon, 2009 : 3). Dit autrement, le paradoxe insurmontable de la littérature interactive consiste à parvenir à octroyer un pouvoir d'action important à l'interacteur sur le récit sans affaiblir symétriquement la narration. Dès lors, le défi du design d'un récit interactif est de parvenir «à faire tenir ensemble narrativité et interactivité » (ibid.). En suivant ce raisonnement et dans l'objectif de préciser les modalités d'action de l'interacteur sur le 
contenu multimédia, différents régimes d'interactivité peuvent être définis. L'axe de pertinence retenu consiste à ainsi qualifier les opérations textuelles qui sont attendues de la part de l'utilisateur. Cette approche permet de discerner quatre niveaux d'interactivité, librement combinables entre autre eux au cours de la consultation d'un documentaire interactif :

11 - Une interactivité de navigation consiste à accéder à un contenu en consultant des unités d'information structurées de manière plus ou moins fragmentaire et arborescente.

12 - Une interactivité de manipulation réside dans le fait de modifier la forme ou l'aspect du même contenu médiatique (notamment à travers la grande plasticité des interfaces).

13 - Une interactivité contributive se caractérise par l'introduction de données de la part de l'interacteur, sans que celle-ci altère de manière significative le contenu éditorial et la forme esthétique du document.

14 - Une interactivité participative se définit par une écriture du lecteur qui altère la forme et/ou la proposition éditoriale du web-documentaire.

15 En résumé, la dialectique de la posture spectatorielle caractéristique du format webdocumentaire oscille entre une nature médiatique propre aux hypermédias (Web) et celle caractéristique des cybermédias (jeux vidéo) comme le synthétise le tableau ci-dessous.

\begin{tabular}{|l|l|l|}
\cline { 2 - 3 } & \multicolumn{2}{|l|}{$\begin{array}{l}\text { D i a l e c t i q u e in t e r a c t i o n n e lle } \\
\text { du web-documentaire }\end{array}$} \\
\hline Nature médiatique & Hypermédia & Cybermédia \\
\hline \multirow{2}{*}{ Logique relationnelle } & $\begin{array}{l}\text { Réticulaire avec } \\
\text { a c t i o n d e } \\
\text { manipulation }\end{array}$ & $\begin{array}{l}\text { Vé h i c u l a i r e a v e c } \\
\text { immersion dans un flux } \\
\text { de données }\end{array}$ \\
\hline \multirow{2}{*}{$\begin{array}{l}\text { Fréquence } \\
\text { d'intervention }\end{array}$} & Séquentielle & Permanente \\
\hline \multirow{2}{*}{$\begin{array}{l}\text { Type d'action sur le } \\
\text { dispositif et régime } \\
\text { d'interactivité associé }\end{array}$} & $\begin{array}{l}\text { Manipuler/ } \\
\text { interactivité de manipulation }\end{array}$ \\
\cline { 2 - 3 } & $\begin{array}{l}\text { Écriture sans altération sur l'œuvre/ } \\
\text { interactivité de visionnage }\end{array}$ \\
\cline { 2 - 3 } & $\begin{array}{l}\text { Écriture avec altération sur l'œuvre/ } \\
\text { interactivité participative }\end{array}$ \\
\hline
\end{tabular}

Tableau : Posture spectatorielle du web-documentaire.

\section{Typologie des « modes d'interaction » du documentaire interactif}

Dans le champ des études cinématographiques, le chercheur américain Bill Nichols (2010) propose de catégoriser l'espace hétérogène du documentaire suivant une typologie des «modes de représentations du réel ». Cette approche communicationnelle et systémique 
vise à questionner la relation triadique entre le cinéaste, le réel et le spectateur ${ }^{11}$. Dans la continuité de ces travaux, Sandra Gaudenzi (2013) propose une ébauche de catégorisation du documentaire interactif basée sur des « modes d'interaction ». Cette typologie consiste désormais à définir des modalités d'interactivité qui positionnent l'interacteur face au document. L'enjeu réside ici dans l'interrogation des spécificités de la médiation entre le dispositif médiatique et le « réel » qu'il dépeint. Dit autrement, il s'agit de questionner la relation systémique entre quatre entités : l'instance d'énonciation, le réel, le dispositif médiatique et l'interacteur. Ce nouveau paradigme reformule le "point de vue documenté » d'un auteur qui consiste - dans le cadre d'un documentaire interactif - à raconter le monde à travers une écriture singulière des "modes d'interaction». Cet article propose de reformuler les modes conceptualisés par Gaudenzi ${ }^{12}$ afin d'établir une typologie du documentaire interactif en six catégories: hypermédiatique, dialogique, contributif, participatif, ludique et performatif. En d'autres termes, il s'agit d'interroger l'état actuel de la production à partir d'une série de questions: Quels sont le pouvoir et les possibilités d'action octroyés à l'interacteur par le dispositif médiatique? Quelle relation entretient l'instance d'énonciation avec les autres actants? Est-il possible de penser un document interactif qui évolue de manière dynamique en fonction du contexte et des usages?

\section{Mode d'interactivité hypermédiatique}

Cette scénarisation de l'interactivité exploite le principe générique des écritures arborescentes qui structurent l'agencement des données selon une quête informationnelle que le lecteur oriente. Jean-Pierre Balpe définit un hyperdocument ${ }^{13}$ comme un "contenu informatif constitué d'une nébuleuse de fragments dont le sens se construit, au moyen d'outils informatiques, à travers chacun des parcours que la lecture détermine » (1990: 6). Selon cette terminologie, un document hypermédia constitue « un ensemble d'informations appartenant à plusieurs types de médias (texte, son, image, logiciels) pouvant être lu (écouté, vu) suivant de multiples parcours de lectures, en utilisant également la possibilité de multi-fenêtrage. Ce qui différencie essentiellement l'hypermédia de l'hypertexte n'est ainsi que la nature symbolique des codages d'information utilisés » (ibid.: 19). Dans cette optique, tout web-documentaire peut être considéré comme un documentaire hypermédia, c'est-à-dire comme un "hypertexte gérant des "textes" supportés par des médias divers" (ibid.). L'intérêt heuristique de cette approche ontologique du web-documentaire est de postuler un lecteur dont le rôle principal est d'explorer ou non une multitude de parcours de lecture virtuellement possibles. Jean-Pierre Balpe souligne néanmoins qu'il n'y a jamais pour chaque lecteur " une simultanéité totale des informations présentes dans l'hyperdocument consulté, dont une grande partie, de ce fait, n'a d'existence que virtuelle » (ibid. : 47).

En outre, il serait aporétique de chercher à définir le web-documentaire comme l'unique extension d'un film documentaire linéaire sous une forme informatisée et connectée. Sa spécificité hypermédiatique le définit ontologiquement comme un objet d'une autre nature, marqué fondamentalement par une modularité et une variabilité. Sa dimension modulaire réside dans le fait que le documentaire hypermédia est créé à l'aide d'éléments indépendants tout en étant reliés les uns aux autres, alors que sa variabilité implique qu'il puisse évoluer dans sa forme et dans sa temporalité. Selon Lev Manovich, à la différence du texte filmique, il est possible de distinguer les différentes entités d'un documentaire 
hypermédia de son architecture informationnelle. "Chaque structure de liens hypertextes peut ainsi être précisée indépendamment du contenu médiatique du document $(2010: 119)$. Le choix de l'architecture hypermédia définit donc un principe de navigation intimement lié à la narration. Quelle que soit la complexité de la structure de l'information (linéaire, arborescente, parallèle, réticulaire, hybride, etc.), l'enjeu pour l'instance d'énonciation consiste à élaborer une multitude de parcours potentiels dans une architecture clôturée. Voyage au bout du charbon (2008), est un exemple emblématique de web-documentaire qui propose un récit à choix multiples sur le modèle bien connu de la littérature dont on est le héros. L'utilisateur est ainsi invité à s'identifier à la figure imaginaire d'un grand reporter indépendant qui mène une investigation journalistique périlleuse sur les conditions de travail des mineurs chinois ${ }^{14}$. Au cours de cette enquête, un nœud dramaturgique lui impose de retourner vers le bureau du directeur de la mine qui le contraint à rebrousser chemin jusqu'à Pékin et à renoncer à l'accomplissement de son projet initial (cf. figure 1). Ce ressort dramatique, emprunté aux scénarios des jeux vidéos d'aventure, simule un court instant une relation dialogique et déplace la relation univoque homme-machine. Au vu de l'état actuel de la production du web-documentaire, force est de constater que le mode d'interactivité hypermédiatique est très largement représenté. Toutefois, ce design de l'interactivité présente deux limites principales. D'une part, il sous-évalue fréquemment les efforts cognitifs nécessaires à l'utilisateur pour construire son parcours de lecture, et d'autre part il n'exploite nullement les potentialités de calcul offerte par les médias informatisés.

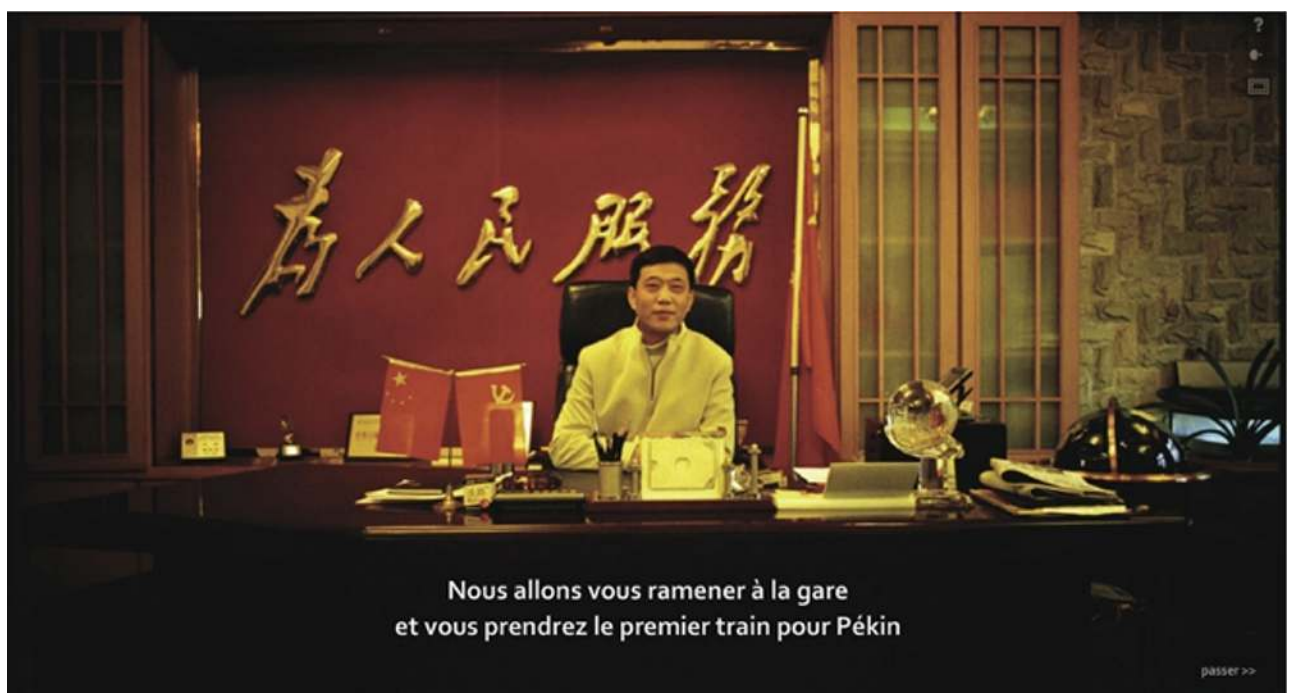

Figure : Capture écran Voyage au bout du Chardon, bureau du directeur.

\section{Mode d'interactivité dialogique}

19 Ce mode consiste à simuler un dialogue entre le dispositif médiatique et l'interacteur selon des règles et des possibilités d'action préétablies. L'utilisateur est ainsi immergé dans un flux de données avec lesquels il peut interagir en temps réel. Cette scénarisation de l'interactivité, plus complexe techniquement à mettre en œuvre que le mode hypermédia, représente l'idéal type d'une interaction véhiculaire proposée par les cybermédias. L'une des productions les plus abouties selon ce critère est probablement le web-documentaire Bear 71 (2012) qui propose une immersion dans l'espace tridimensionnel du parc naturel Banff situé dans les rocheuses canadiennes ${ }^{15}$. La 
représentation symbolique du paysage sculpte un territoire constitué de collines, vallons, forêts et parcouru par de multiples cours d'eau, voies ferrées et autoroutes. Le traitement graphique et symbolique est stylisé par des points, croix, hachures et autres motifs géométriques. La perception du décor est mouvante et suit le fil du déplacement d'un avatar à la recherche des traces laissées par le passage d'une femelle grizzli (Bear 71) équipée d'un émetteur GPS. Au cours de sa déambulation, l'utilisateur peut visionner une série d'images filmées par les caméras de surveillance du parc qui attestent du passage de la faune sauvage (cerf, renard, ours, rapaces, etc.) et de la présence de quelques touristes à différents moments du jour et de la nuit et différentes saisons (cf. figure 2). De plus, si l'utilisateur l'autorise, le site réintègre le flux de sa webcam dans la diégèse du webdocumentaire. Cette captation audiovisuelle est mise en scène au sein d'une interface multi-écrans représentant le flux des images des différentes caméras de vidéo surveillances (cf. figure 3). L'utilisateur est par conséquent mis en abîme dans le dispositif médiatique auquel il participe tout en l'alimentant. Il devient l'observateur de sa propre présence au sein de l'ensemble de la faune qui déambule dans cet espace sauvage.

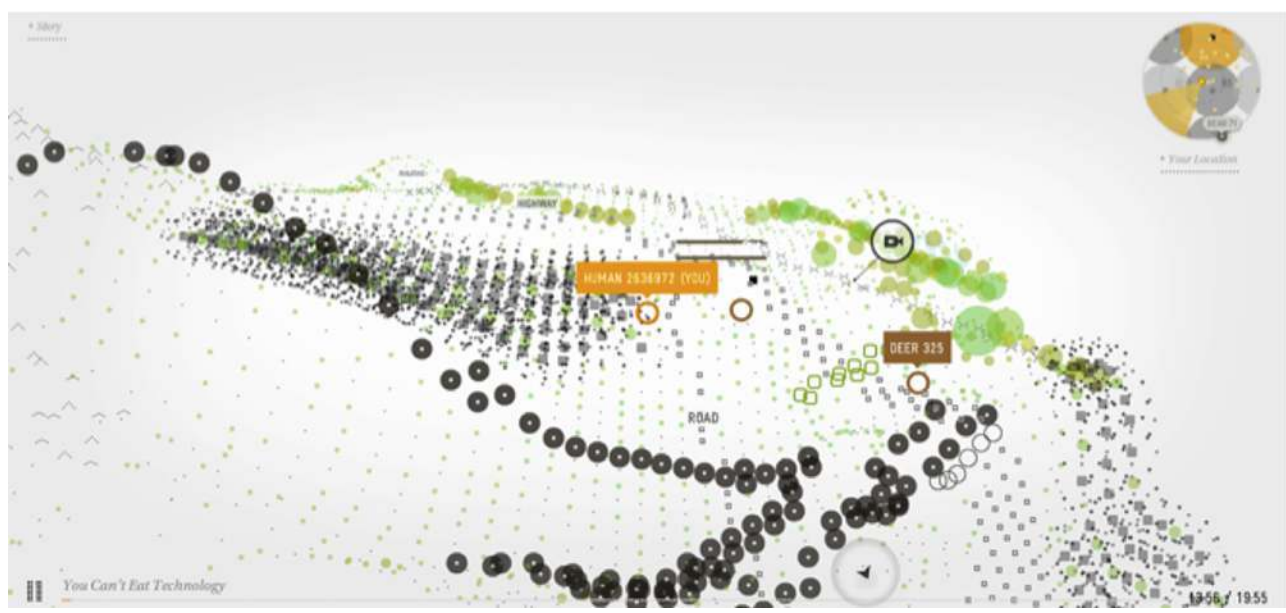

Figure : Capture écran Bear 77, espace interactif tridimensionnel.

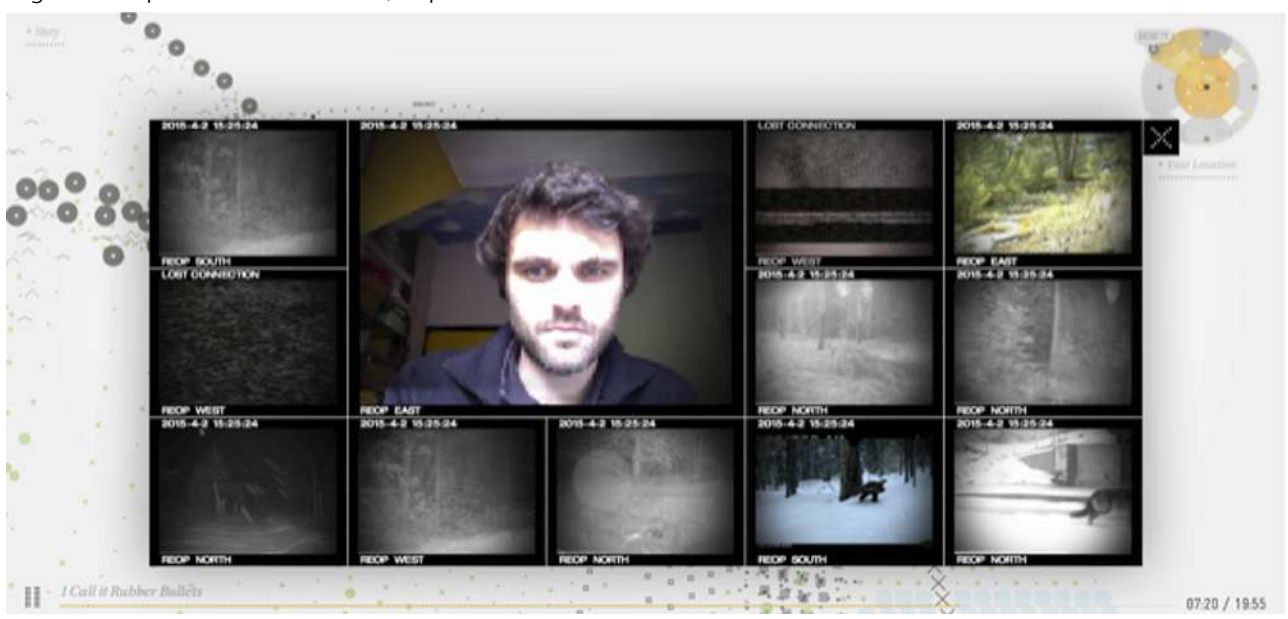

Figure : Capture écran Bear 71, web-cam de l'utilisateur intégrée à l'interface-film.

\section{Mode d'interactivité contributif}

Ce mode est exploité par un très grand nombre de web-documentaires qui proposent à l'interacteur de participer à l'expérience en postant un contenu éditorial. L'enjeu 
auctorial consiste ici à scénariser un cadre de médiatisation afin de susciter une contribution sans altérer la diégèse et le webdesign de l'œuvre. Dans le contexte marketing et idéologique du «Web 2.0 ", toute œuvre interactive sur le réseau semble devoir octroyer une capacité d'intervention à l'utilisateur. Les fonctionnalités proposées à l'internaute ne doivent plus uniquement permettre d'accéder ou de manipuler un contenu préexistant, mais inciter l'utilisateur à introduire ses propres données qui seront ensuite intégrées à l'interface-film. Suivant ce raisonnement, le web-documentaire peut être entendu simultanément comme une machine et un mécanisme "dans le sens où il aménage différentes prises en direction d'un public qui peut désormais, selon certaines réserves et conditions, devenir acteur de fragments de l'œuvre préalablement identifiés " (Fourmentraux, 2010: 39-40). A titre d'exemple, Code Barre (2011) invite l'utilisateur à consulter une base de données de cent portraits d'objets réalisés par trente réalisateurs. L'internaute peut également contribuer au web-documentaire en postant la photographie d'un objet qu'il légende alimentant par là même une bibliothèque d'objets librement consultables en ligne (cf. Figure 4).

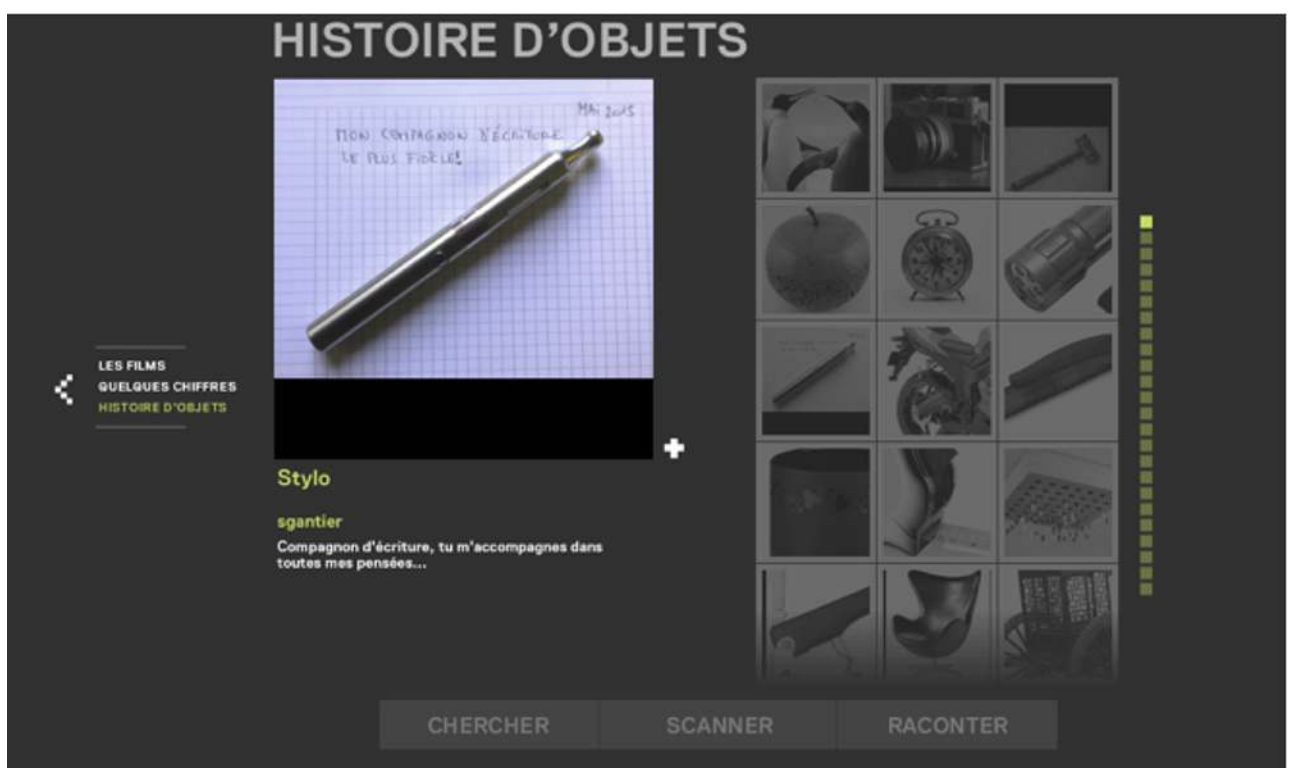

Figure : Capture écran Code Barre, espace contributif « Raconter un objet ».

21 Si l'intérêt principal de ce mode d'interactivité est d'actualiser le contenu du site pendant une période donnée, sa mise en œuvre soulève de nombreuses questions : Jusqu'où peuton déléguer à n'importe quel internaute le pouvoir d'écrire ce qu'il veut sur l'interface du web-documentaire? Comment concilier une ambition de contenu de qualité tout en mobilisant la participation la plus large possible? De quelle manière agencer les contributions des internautes de façon élégante sans dénaturer la proposition graphique du site ? Dès lors, on retrouve à travers cette fonctionnalité une tension dialectique entre le souhait de favoriser une «libre expression » et la volonté de conserver le contrôle éditorial du site si bien que l'instance d'énonciation doit généralement penser une médiation supplémentaire entre l'œuvre et son public. Du point de vue de la production, il s'agit fréquemment de financer un poste de community manager après la mise en ligne, ou de développer un protocole technique de gestion des données qui puisse être automatisé pendant toute la durée de vie du web-documentaire.

22 En outre, loin d'être un phénomène social généralisé comme le vantent les promoteurs du «Web 2.0 », la création de contenu sur Internet reste une activité socialement 
discriminée qui ne rassemble pas toute la diversité des profils composant une société. Selon Franck Rebillard, ces publications sont "principalement l'œuvre d'une minorité sociale, celle qui d'ores et déjà exerçait des activités en lien avec l'information et la culture avant l'arrivée d'Internet » (2007: 51). D'après ce chercheur, il ne suffit pas que les individus soient armés intellectuellement et techniquement pour que la création de contenus sur le Web devienne un usage socialement stabilisé car « leur passage à l'acte est avant tout tributaire de l'environnement macro-social dans lequel ils évoluent» (ibid.: 55). Ainsi, seul le contexte peut permettre à des pratiques pionnières de ne pas rester éphémères et de se transformer, sous la forme d'habitudes intégrées dans la quotidienneté, afin de résister en tant que pratiques spécifiques, de s'imposer ou de cohabiter avec des pratiques culturelles préexistantes.

\section{Mode d'interactivité participatif}

Ce mode est celui qui déplace le plus l'instance auctoriale vers le pôle de la réception dans la mesure où il attribue un rôle déterminant à de nouvelles instances d'énonciations documentaires. En favorisant la participation active des protagonistes à l'écriture de leur propre représentation, ce mode de scénarisation de l'interactivité ouvrirait la possibilité d'octroyer un rôle plus important à de nouvelles « voix documentaires »16. Autrement dit, il s'agit de permettre la prise de parole de ceux qui auparavant étaient formellement désignés comme les sujets du documentaire. Ce constat amène William Uricchio, à considérer que :

le documentaire est à nouveau en train d'ouvrir de nouvelle voies de représentation et d'intervention dans le monde. Mais cette fois, au lieu de simplement recourir à de nouvelles techniques pour représenter le changement social, c'est la forme documentaire elle-même [je souligne] qui est le sujet des transformations sociales et technologiques. (2014: 61).

Le web-documentaire américain Sandy Storyline (2012) propose par exemple aux habitants touchés par l'ouragan de mettre en récit leurs propres témoignages sur la manière dont ils ont intimement vécu cette catastrophe naturelle (cf. figure 5). Dans un tout autre contexte, 18 Days in Egypt (2011) relate les événements politiques de la révolution égyptienne à travers le point de vue des anonymes qui y ont directement participé. Suivant ce raisonnement, le mode participatif représenterait

le changement le plus significatif en termes de forme, de mode d'adresse et de relation au public depuis le cinéma direct et le cinéma vérité [...]. Le documentaire, longtemps identifié à une forme, à ce média linéaire qu'est le film ou la télévision (média de flot), [renaitrait] en tant que mission comme un mode d'engagement et d'interaction incroyablement agnostique en ce qui concerne sa forme (Uricchio, 2014 : 66-68).

Plus généralement, cette perspective pour l'émergence d'une poḯtique documentaire renvoie à la notion d'empowerment de l'utilisateur développée notamment dans le champ du design de produits et services. ${ }^{17}$ 


\section{Featured}

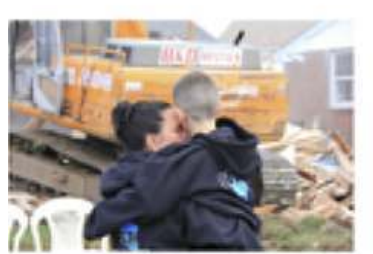

Unien Beach 2 Years Later

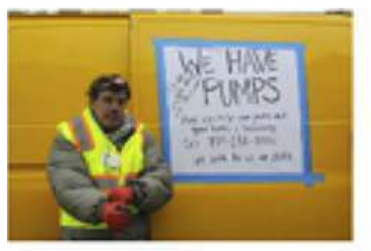

Responding in Rockaway

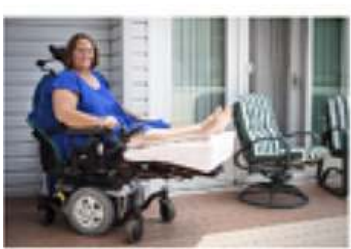

12 Menths - Mary Elles Olsen

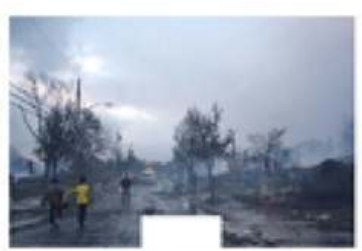

Fire on seach 130th

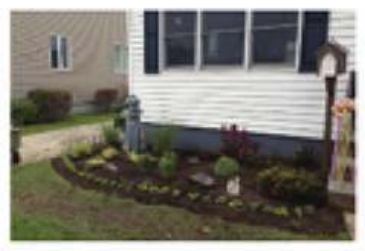

Finally Made It Home

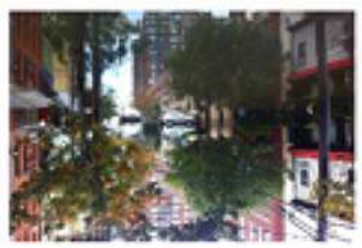

The Eeautiful Thing in Life
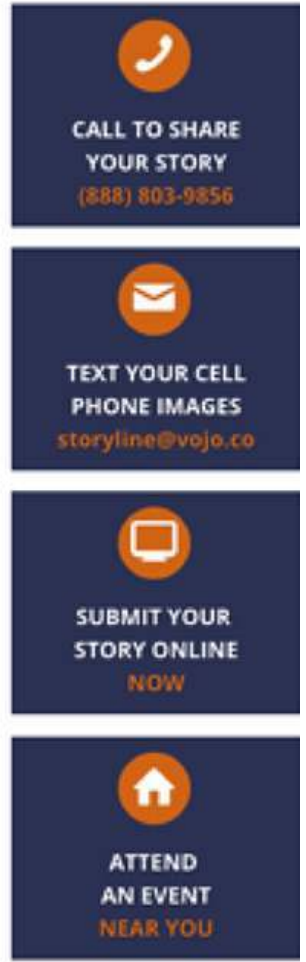

Figure : Capture écran Sandy Storyline, dispositif participatif.

\section{Mode d'interactivité ludicisant}

Ce procédé vise à mettre en place un dispositif de ludification (gamification) du récit. L'enjeu pour l'instance auctoriale consiste désormais à élaborer la jouabilité du documentaire interactif ${ }^{18}$. Ce mode d'interactivité part du postulat que pour améliorer l'engagement de l'interacteur dans la narration documentaire, il est pertinent et efficient de déployer divers mécanismes de récompense (points, médailles, niveaux). En d'autres termes, cette approche promeut une boucle « challenge-réussite-récompense » à travers des processus de rétroaction supposés procurer du plaisir à l'utilisateur. L'une des principales critiques suscitées par cette notion dans le champ académique des game studies porte sur la pauvreté du game design ${ }^{19}$ qu'induit cette approche résolument behaviouriste. En effet, un jeu n'est pas intéressant simplement parce qu'il est un jeu mais avant tout parce qu'il est bien conçu. L'adjonction d'un système de points ou de récompenses ne peut bien évidemment suffire à traduire n'importe quelle démarche documentaire en un jeu qui documente le réel de manière intéressante. Afin de se distancier de la forte influence marketing qui caractérise cette notion, Sébastien Genvo propose de nommer « ludicisation ${ }^{20}$ » les « processus qui consistent à faire entrer un objet dans l'aire du jeu, [...] en soulignant que l'acception prêtée à ce terme est relative à un certain contexte socio-culturel» (2013: 41). Ainsi nombre de web-documentaires se donnent pour projet de susciter une attitude ludique à travers différentes mécaniques plus ou moins élaborées. Dans cette optique ces productions intercalent des jeux simples et courts au sein du contenu dit sérieux. Déclinant le modèle des serious game qui ciblent des joueurs occasionnels (casual game), Amour 2.0 (2012) remobilise par exemple la culture des quizz tel que les diffuse la «presse féminine ». Chaque questionnaire s'intercale ainsi 
entre les différents modules documentaires qui composent le récit hypermédia (cf. figure 6). De manière plus élaborée, Fort McMoney (2013) est le premier documentaire interactif à revendiquer l'appellation de «jeu documentaire». Ce web-documentaire multi-joueurs propose un game design directement inspiré de l'illustre jeu vidéo SimCity ${ }^{21}$. Le but du jeu consiste à prendre possession de la troisième réserve de pétrole mondiale afin de débattre de la place de cette énergie dans notre société. Pour ce faire, le webdesign mobilise un ensemble de codes issus de la culture des jeux vidéo : tableau de bord qui attribue des responsabilités aux joueurs à l'image des jeux d'aventures (cf. figure 7) ; compteur de voiture dont l'aiguille oscille comme dans un jeu de course automobile; point de vue subjectif du narrataire pendant les séquences interactives renvoyant aux «jeux de tir à la première personne ${ }^{22}$ »; « fenêtre inventaire » où le joueur peut stocker les objets collectés à la manière des jeux de rôle ${ }^{23}$; etc.

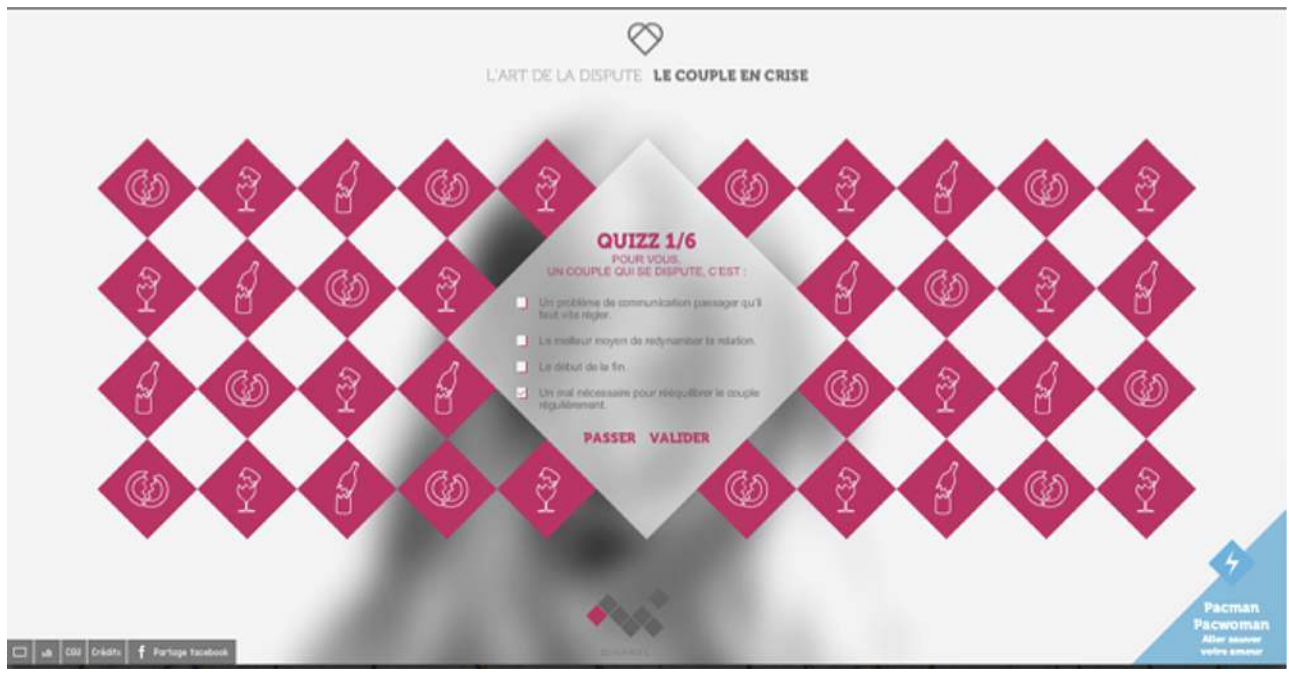

Figure : capture d'écran Amour 2.0, Quizz 1/6.

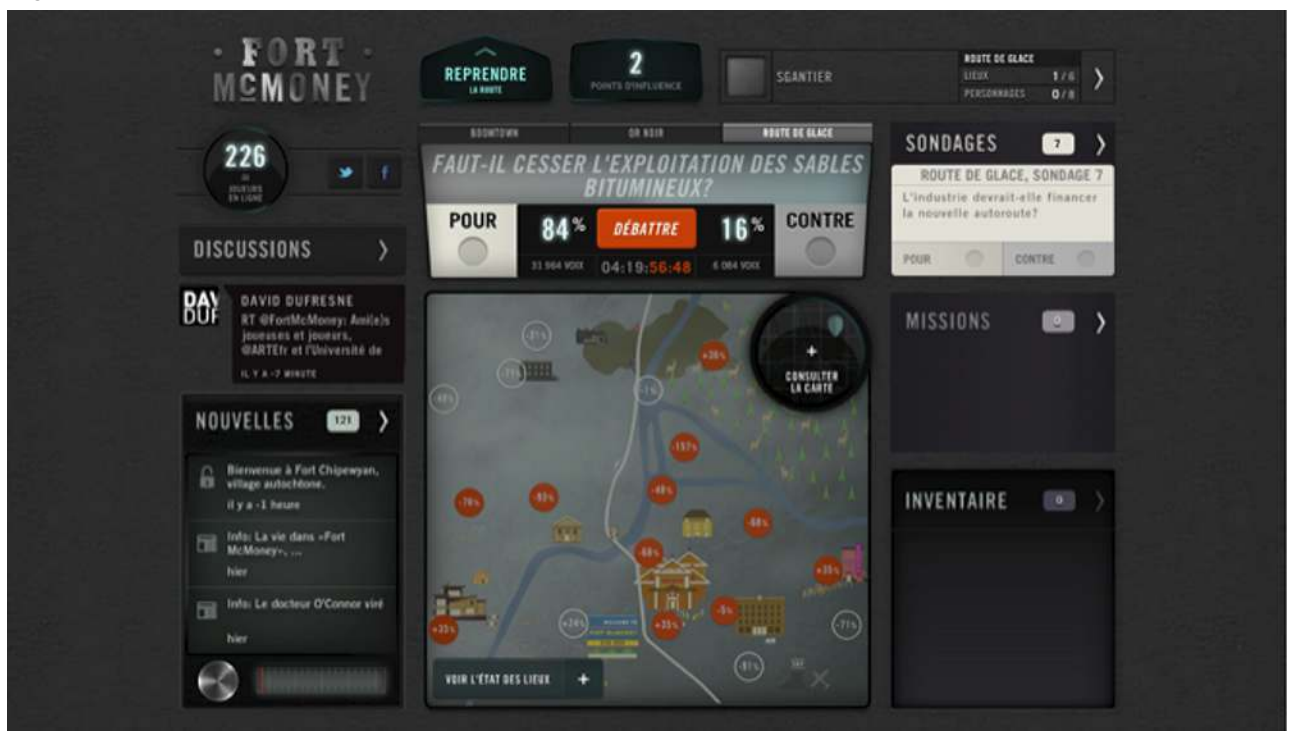

Figure 7 : Capture écran Fort McMoney, tableau de bord. 


\section{Mode d'interactivité performatif}

Ce design d'interaction cherche à exploiter les dimensions ubiquitaires et connectées des terminaux mobiles. Il s'agit d'une écriture performative dont l'objectif est d'interagir avec le réel à travers les potentialités offertes notamment par les médias géolocalisés et la « réalité augmentée ${ }^{24}$ ». Aujourd'hui, peu de productions parviennent à tirer pleinement parti de ce mode d'interactivité complexe et ambitieux. À titre d'exemple, Jaurès pas à pas (2014) est un documentaire interactif qui propose des parcours thématiques et historiques dans les villes de Paris et Toulouse afin de débloquer une série de modules documentaires sur la vie de Jean Jaurès (cf. figure 8 ). Il va de soi que le développement de ce type d'écriture in situ nécessite un budget relativement important ramené au nombre d'utilisateur pouvant participer à l'expérience sur un territoire donné. Ce mode d'interactivité positionne toutefois la création documentaire interactive au carrefour de la muséographie et de la valorisation patrimoniale du territoire.

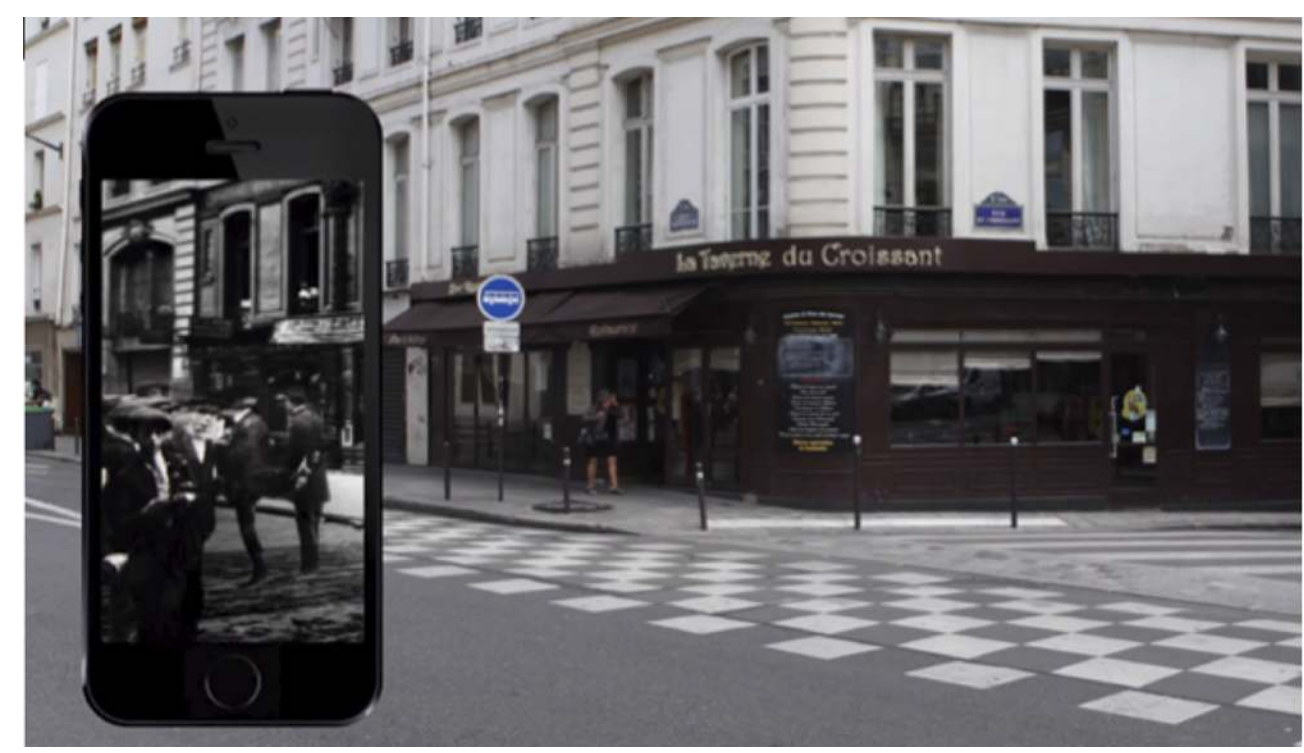

Figure : Extrait de la bande annonce Jaurès pas à pas

\section{Conclusion}

Au vu de l'état actuel de la production, cette typologie interactionnelle du documentaire interactif met en exergue le potentiel créatif des «nouvelles écritures» du réel. Les spécificités du design d'interaction soulignent la dimension relationnelle entre interacteur et instance auctoriale en déclinant une série de six modes d'interaction librement combinables entre eux (cf. tableau ci-dessous). Toutefois, face au caractère labile et non stabilisé du documentaire interactif, une prudence épistémologique s'impose. En effet, les six modes exposés précédemment ne sont ni exclusifs ni exhaustifs. Une grande partie des publications de web-documentaires francophones combinent plusieurs modes d'interaction simultanément. D'autre part, des innovations récentes, notamment à travers la visualisation de données complexes ou la scénarisation de récit génératif qui exploitent des bases de données d'archives audiovisuelles ${ }^{25}$ ouvrent la voie à de nouvelles expérimentations qui peuvent à l'avenir reformuler cette proposition 
provisoire de classification. En d'autres termes, chaque classement renvoie aux enjeux épistémologiques de leur fabrication et aucune typologie ne peut prétendre être exhaustive et définitive. Comme le prétend Guy Gauthier si «la carte n'est pas le territoire, [elle permet néanmoins] de s'y retrouver si elle est construite par des conventions clairement explicitées » $(1995: 186)$.

\begin{tabular}{|c|c|c|c|c|}
\hline $\begin{array}{l}\text { Modes } \\
\text { d'interaction }\end{array}$ & $\begin{array}{l}\text { Structure du } \\
\text { texte } \\
\text { multimédia }\end{array}$ & $\begin{array}{l}\text { Travail de } \\
\text { conception }\end{array}$ & $\begin{array}{l}\text { Travail de } \\
\text { réception }\end{array}$ & $\begin{array}{l}\text { Exemples } \\
\text { emblematiques }\end{array}$ \\
\hline $\begin{array}{l}\text { Hypermédia } \\
\text { tique }\end{array}$ & $\begin{array}{l}\text { Arborescent } \\
\text { et clôturé }\end{array}$ & $\begin{array}{l}\text { Construire une } \\
\text { multitude de } \\
\text { parcours de lecture } \\
\text { dans une } \\
\text { architecture } \\
\text { fermée. }\end{array}$ & $\begin{array}{l}\text { Choisir un } \\
\text { parcours de } \\
\text { lecture } \\
\text { singulier. }\end{array}$ & $\begin{array}{l}\text { Voyage au bout } \\
\text { du charbon } \\
(2008)\end{array}$ \\
\hline Dialogique & $\begin{array}{l}\text { Flux de } \\
\text { données } \\
\text { actualisé en } \\
\text { temps réel }\end{array}$ & $\begin{array}{l}\text { Simuler un } \\
\text { dialogue selon des } \\
\text { régles et des } \\
\text { possibilités } \\
\text { d'action } \\
\text { prététablies. }\end{array}$ & $\begin{array}{l}\text { Interagir avec } \\
\text { l'interface-film } \\
\text { en restant } \\
\text { immergée dans } \\
\text { une diégèse. }\end{array}$ & $\begin{array}{l}\text { Bear 71 } \\
\text { (2012) }\end{array}$ \\
\hline Contributif & $\begin{array}{l}\text { Actualisable } \\
\text { et semi- } \\
\text { ouvert }\end{array}$ & $\begin{array}{l}\text { Scénariser un } \\
\text { cadre de } \\
\text { médiatisation afin } \\
\text { de susciter une } \\
\text { contribution sans } \\
\text { altérer l'cusve. }\end{array}$ & $\begin{array}{l}\text { Lecture et/ou } \\
\text { entrée de } \\
\text { données dans le } \\
\text { dispositif. }\end{array}$ & $\begin{array}{l}\text { Code Barre } \\
\text { (2011) }\end{array}$ \\
\hline Participatif & $\begin{array}{l}\text { Actualisable } \\
\text { et ouvert }\end{array}$ & $\begin{array}{l}\text { Scénariser un } \\
\text { cadre de } \\
\text { médiatisation afin } \\
\text { de susciter une } \\
\text { participation avec } \\
\text { altération de } \\
\text { l'ceuvre. }\end{array}$ & $\begin{array}{l}\text { Lecture et } \\
\text { entrée de } \\
\text { données dans le } \\
\text { dispositif. }\end{array}$ & $\begin{array}{l}\text { Sandy Storyline } \\
\text { (2012) }\end{array}$ \\
\hline Ludicisant & Jouable & $\begin{array}{l}\text { Elaborer le game } \\
\text { design et game } \\
\text { play du document. }\end{array}$ & $\begin{array}{l}\text { Opter pour une } \\
\text { attitude ludique } \\
\text { afin } \\
\text { d'expérimenter } \\
\text { la jouabilité du } \\
\text { document. }\end{array}$ & $\begin{array}{l}\text { Fort McMoney } \\
\text { (2013) }\end{array}$ \\
\hline Performatif & Géolocalisé & $\begin{array}{l}\text { Définit la relation } \\
\text { entre les médias } \\
\text { interfacés et le } \\
\text { cadre pragmatique } \\
\text { de la consultation. }\end{array}$ & $\begin{array}{l}\text { Perçoit une } \\
\text { réalité } \\
\text { «augmentée } " \\
\text { à travers sa } \\
\text { manipulation in } \\
\text { situ des médias }\end{array}$ & $\begin{array}{l}\text { Jaurès } \\
\text { pas à pas } \\
\text { (2014) }\end{array}$ \\
\hline
\end{tabular}

Tableau : Typologie du design d'interaction du web-documentaire

31 Bien que cette typologie du design d'interaction du web-documentaire permette de révéler les spécificités du travail de conception et de réception, il ne faudrait pas perdre de vue que l'interactivité reste un outil technique et conceptuel au service du point de vue d'un auteur. La praxis documentaire réside en effet dans une pratique artistique qui se donne pour objet de déconstruire le réel afin de rendre visibles des phénomènes invisibles autrement à notre entendement. Autrement dit, le projet politique et esthétique d'une démarche documentaire - interactive ou non - consiste à déconstruire des phénomènes réels pour permettre au spectateur d'accéder à une compréhension de processus complexes sous-jacents qui échappent à la perception immédiate. Ce dessein du design du web-documentaire permet de repositionner les innovations technologiques (telles que la réalité augmentée, la géolocalisation ou la visualisation de données complexes) comme des opportunités d'écritures innovantes, nécessaires, mais jamais suffisantes pour permettre l'émergence d'une nouvelle esthétique documentaire. En outre, le modèle théorique du design du documentaire interactif présenté dans cet article reste une conceptualisation des œuvres détachée de leurs conditions écologiques de production et de réception. Pour permettre de saisir pleinement les évolutions en cours depuis quelques années dans le champ des industries culturelles, ces hypothèses doivent être mises à l'épreuve du terrain. En effet, seule une évaluation de l'expérience utilisateur permettrait de confronter le projet idéal du design du web-documentaire à l'appropriation du dispositif par l'utilisateur. 


\section{BIBLIOGRAPHY}

Balpe Jean-Pierre, 1990, Hyperdocuments, Hypertextes, Hypermédias, Paris, Eyrolles.

Bolka-Tabary Laure et Gantier Samuel, 2011, « L'expérience immersive du web documentaire : études de cas et pistes de réflexion », Les Cahiers du journalisme, no 22-23, pp. 118-133[en ligne : http://www.cahiersdujournalisme.com/ cdj/pdf/22_23/08_BOLKA_GANTIER.pdf.]

Bouchardon Serge, 2009, Littérature numérique : le récit interactif, Paris, Hermès/Lavoisier.

Di Crosta Marida, 2009, Entre cinéma et jeux vidéo : l'interface-film, Métanarration et interactivité, Bruxelles/Bry-sur-Marne, De Boeck/INA.

Fourmentraux Jean-Paul, 2010, Art et Internet : les nouvelles figures de la création, Paris, CNRS éditions.

Gantier Samuel et Labour Michel, « User Empowerment and the I-Doc Model User », in User Empowerment: Interdisciplinary studies \& combined approaches for technological products and services, Bihanic D. (dir.), Springer Verlag, Londres, janvier 2015, pp. 231-254.

Gantier Samuel, 2012, «Le webdocumentaire, un format hypermédia innovant pour scénariser le réel ? », dans Journalisme en ligne, Bruxelles, De Boeck, pp. 159-177.

Gaudenzi Sandra, 2013, The Living Documentary : From representing reality to co-creating reality in digital interactive documentary, thèse de doctorat, Goldsmiths (Centre for Cultural Studies), Université de Londres.

Gauthier Guy, 1995, Le documentaire : un autre cinéma, Paris, Armand Colin.

Genvo Sébastien, 2013, Penser la formation et les évolutions du jeu sur support numérique, mémoire pour l'habilitation à diriger des recherches en sciences de l'information et de a communication, université de Lorraine.

Guéneau Catherine, 2005, «L'interactivité : une définition introuvable », Communication et langages, vol. 145, no 1, pp. 117-129.

Jeanneret Yves, 2007, Y a-t-il (vraiment) des technologies de l'information ?, Villeneuve-d'Ascq, Presses du Septentrion.

Jeanneret Yves et Souchier Emmanuël, 1999, « Pour une poétique des écrits d'écran », Xoana, 1999, no 6-7, pp. 97-107.

Julia Jean-Thierry, 2003, « Interactivité, modes d'emploi : réflexions préliminaires à la notion de document interactif », Documentaliste - Sciences de l'Information, vol. 40, no 3, pp. 204-212.

Manovich Lev, 2010, Le Langage des nouveaux médias, Dijon, Les Presses du réel.

Nash Kate, 2014, «Cliking on the World : Documentary Representation and Interactivity », dans K. Nash, C. Hight et C. Summerhayes (dirs.), New Documentary Ecologies : Emerging Platforms, Practices and Discourses, New York, Palgrave Macmillan, pp. 50-66.

Nichols Bill, 2010, Introduction to documentary, Bloomington, Indiana University Press. 
Perény Étienne et Amato Étienne Armand, 2011, « Audiovisuel interactif », Communications, no 1, pp. 29-36.

Uricchio William, 2014, « Repenser le documentaire social », dans L. Allard, L. Creton et R. Odin

(dirs.), Téléphone mobile et création, Paris, Armand Colin, pp. 61-79.

\section{RESSOURCES WEB}

L'ensemble des URL a été vérifié le 10/05/2015.

18 Days in Egypt (2011), http://beta.18daysinegypt.com/

Amour 2.0 (2012), http://www.francetv.fr/amour/

Bear 71 (2012), http://bear71.nfb.ca/\#/bear71

Code Barre (2011), http://codebarre.tv/fr/\#/fr

Fort McMoney (2013), http://www.fortmcmoney.com/\#/fortmcmoney

Générations 14 (2014), http://generations-14.fr/

L'Obésité est-elle une fatalité ? (2009), http://education.francetv.fr/

webdocumentaire/l-obesite-est-elle-une-fatalite-o22876

Le Challenge (2009), http://www.canalplus.fr/c-infos-documentaires/pid3400-c-le-challenge.html

Sandy Storyline (2012), http://www.sandystoryline.com/

Voyage au bout du charbon (2008), http://www.lemonde.fr/asiepacifique/

visuel/2008/11/17/voyage-au-bout-du-charbon_1118477_3216.html

\section{NOTES}

1. Source : http://www.klynt.net/fr/.

2. L'exploitation commerciale de 3WDOC a cessé en décembre 2013. Source: http:// www.3wdoc.com/fr/.

3. Depuis 2014, Djehouti a été rebaptisé Racontr. Source : http://www.djehouti.com/97djehouti.htm.

4. Si ces logiciels sont les principaux utilisés en France, d'autres outils anglo-saxons existent également comme Zeega, Tumulte Hype ou Pop Corn. Un descriptif de ces technologies est consultable sur le blog web-documentaire.org, http://www.webdocumentaire.org/liens/outils-creation-technologies-webdocumentaires-webdocs.html/.

5. Jeanneret et Souchier définissent les architextes comme des «outils qui permettent l'existence de l'écrit à l'écran et qui, non contents de représenter la structure du texte, en commandent l'exécution et la réalisation. Autrement dit, le texte naît de l'architexte qui en balise l'écriture » (1999: 105-108).

6. Le fonds des " projets pour les nouveaux médias » et la mise en œuvre du " web COSIP» en 2011 par le CNC a favorisé le développement d'œuvres audiovisuelles interactives « patrimoniales » pour le Web. Parallèlement les régions Ile de France, Nord-Pas de Calais et Rhône Alpes notamment ont élargi leur politique d'aide à la création audiovisuelle en finançant des contenus interactifs innovants.

7. Avec l'émergence de la télématique (associée de manière métonymique au déploiement du Minitel en France), le terme d'interactivité apparaît comme le "mot-slogan » pour 
véhiculer l'image de marque des réseaux câblés à la française (cf. Rabaté et Lauraire, 1985 : 17-81).

8. Se reporter à Julia (2003) pour les nombreuses références bibliographiques afférentes à cette revue de littérature abondante.

9. Les recherches de Jean-Louis Weissberg et Pierre Barboza (2006) dans le champ des arts contemporains et du jeu vidéo proposent notamment le concept d'image "actée », qui décrit des œuvres interactives où l'action corporelle est directement l'objet de l'interaction dans un mouvement d'agrégation du geste et du regard. De nombreuses installations proposent ainsi des expériences performatives qui alimentent une dynamique de " gestuelle interfacée ».

10. L'expression de récit littéraire interactif désigne selon Serge Bouchardon un corpus d'œuvres hétérogènes qui ne constitue pas, à proprement parler, un genre durable. L'intérêt heuristique de cet objet réside donc principalement «dans sa faculté d'interrogation : du récit, du dispositif interactif, du multimédia et de la littérature. C'est parce que le récit littéraire interactif est travaillé par un jeu de tensions qu'il a ce pouvoir interrogateur, voire qu'il peut jouer un rôle de révélateur » (Bouchardon, 2009 : 303).

11. Ces différents modes sont des catégories intellectuelles opératoires et observables qui se distinguent des écoles esthétiques historiquement situées. L'auteur souligne que ces « patterns» représentent six idéaux types (exposé, observation, poétique, réflexif, participatif et performatif) qui peuvent se combiner et s'hybrider selon les pratiques des cinéastes (cf. Nichols, 2010 : 142-211).

12. Sandra Gaudenzi établit sa typologie en distinguant quatre modes d'interaction : hypertextuel, conversationnel, participatif et expérimental (2013:21-68).

13. La paternité des concepts d'hypertexte et d'hypermédia est généralement attribuée au sociologue américain Ted Nelson. Celui-ci utilise ces néologismes en 1965 pour décrire son fameux système d'information Xanadu, qui permet théoriquement de partager des données informatiques de manière instantanée et universelle.

14. Pour une analyse plus approfondie de la représentation archétypale du grand reporteur dans les web-documenntaires Voyage au bout du charbon (2008), L'obésité est-elle une fatalité ? (2009) et Le Challenge (2009) voir «L'expérience immersive du web documentaire : études de cas et pistes de réflexion » (Bolka-Tabary et Gantier, 2011).

15. Pour une analyse approfondie de ce web-documentaire voir notamment «Cliking on the world : Documentary Representation and Interactivity " (Nash, 2014).

16. Le concept de "voix documentaire » renvoie aux théories du chercheur américain Bill Nichols. Aucune traduction française de ce concept n'existant dans la littérature francophone à ma connaissance, je propose comme équivalent l'idée "d'instance d'énonciation documentaire ».

17. Le terme d'empowerment se définit comme l'attribution de davantage de pouvoir aux individus ou aux groupes pour agir sur les conditions sociales, économiques, politiques ou écologiques qu'ils subissent. Suivant les contextes, on retrouve différentes traductions en français : capacitation, développement du pouvoir d'agir, autonomisation, responsabilisation, émancipation, pouvoir-faire, etc. Source: http://fr.wikipedia.org/ wiki/Empowerment. Sur les enjeux de cette notion dans le champ du documentaire interactif voir notamment « User Empowerment and the I-Doc Model User» (Gantier et Labour, 2015). 
18. Le concept « d'èthos ludique » précise les modalités d'une structure ludique qui induit que le web-documentaire doit être perçu comme un jeu (cf. Genvo, 2013).

19. Le game design se définit comme le processus de création et de conception des règles et autres éléments constitutifs d'un jeu.

20. Cette théorie communicationnelle de la «ludicisation 》 (Genvo, 2013) s'oppose aux perceptions immanentistes du jeu et propose de penser la médiation des phénomènes ludiques à l'aide de trois concepts : la jouabilité, l'éthos ludique et le joueur modèle.

21. SimCity est une série de jeux vidéo débutée par Will Wright en 1989 pour le compte de la société Maxis. En tant que maire, le joueur doit transformer un terrain vide en agglomérations puis administrer une ville et ses infrastructures à l'aide d'un budget.

22. FPS signifie « First person shooter ».

23. RPG signifie « Role playing game ».

24. La notion de réalité augmentée désigne les systèmes informatiques qui permettent la superposition d'un modèle virtuel 3D ou 2D à la perception que nous avons « naturellement » de la réalité, et ceci en temps réel.

25. Voir notamment le web-documentaire Génération 14 (2014) qui invite l'utilisateur à rechercher la trace de ses aïeux poilus à travers une base de donnée d'archives nationales. Celui-ci a la possibilité de poster ses propres archives qui sont dans un deuxième temps intégrées à un récit documentaire sur la grande guerre.

\section{ABSTRACTS}

During the 2005-2015 decade hundreds of French- and English-speaking Interactive Documentaries (idocs) have been published on the Internet. These documentary "new writings" have profoundly modified the role and place attributed to the user. Given this on what basis can one categorise heterogeneous Interactive Documentaries (idoc)? In response to this question, our paper advances an interactional typology of idoc productions resting on six complementary modes: hypermedia, dialogic, contributive, participative, ludic and performative. In referring to emblematic examples of idoc productions, our categorisation examines the systemic rapport of three entities: enunciation instances, media systems, interactants. The heuristic aim of our typology is twofold. It helps identify the characteristics of the auctorial function. It also analyses the characteristics of users' lived experiences.

Sur la décennie 2005-2015, plusieurs centaines web-documentaires ont été publiés sur Internet. Ces «nouvelle écritures » documentaires modifient en profondeur la place et le rôle attribués à l'interacteur. Selon quels critères peut-on classer l'hétérogénéité de ces écritures documentaires interactives? En filiation avec les théories communicationnelles des "modes d'interaction" (Gaudenzi, 2013), cet article propose une typologie interactionnelle de la production suivant six entrées complémentaires: hypermédiatique, dialogique, contributif, participatif, ludique et performatif. Sur la base d'exemples emblématiques de la production, cette catégorisation interroge la relation systémique entre trois entités : instance d'énonciation, dispositif médiatique et interacteur. L'ambition heuristique de cette taxinomie est double : elle permet d'une part de 
discerner les spécificités du travail auctorial et d'autre part, d'analyser les compétences spectatorielles mobilisées par l'utilisateur.

\section{INDEX}

Mots-clés: web-documentaire, documentaire interactif, design d'interaction, typologie interactionnelle, hypermédia, multimédia, conception, réception.

\section{AUTHOR}

\section{SAMUEL GANTIER}

Maître de conférences associé à l'Université de Valenciennes où il enseigne l'audiovisuel transmédia et le design du web-documentaire. Docteur en sciences de l'information et de la communication et sciences de l'art, sa recherche au sein du laboratoire DeVisu concerne les processus d'innovation dans la conception/réception d'œuvres audiovisuelles interactives pour les nouveaux médias. 